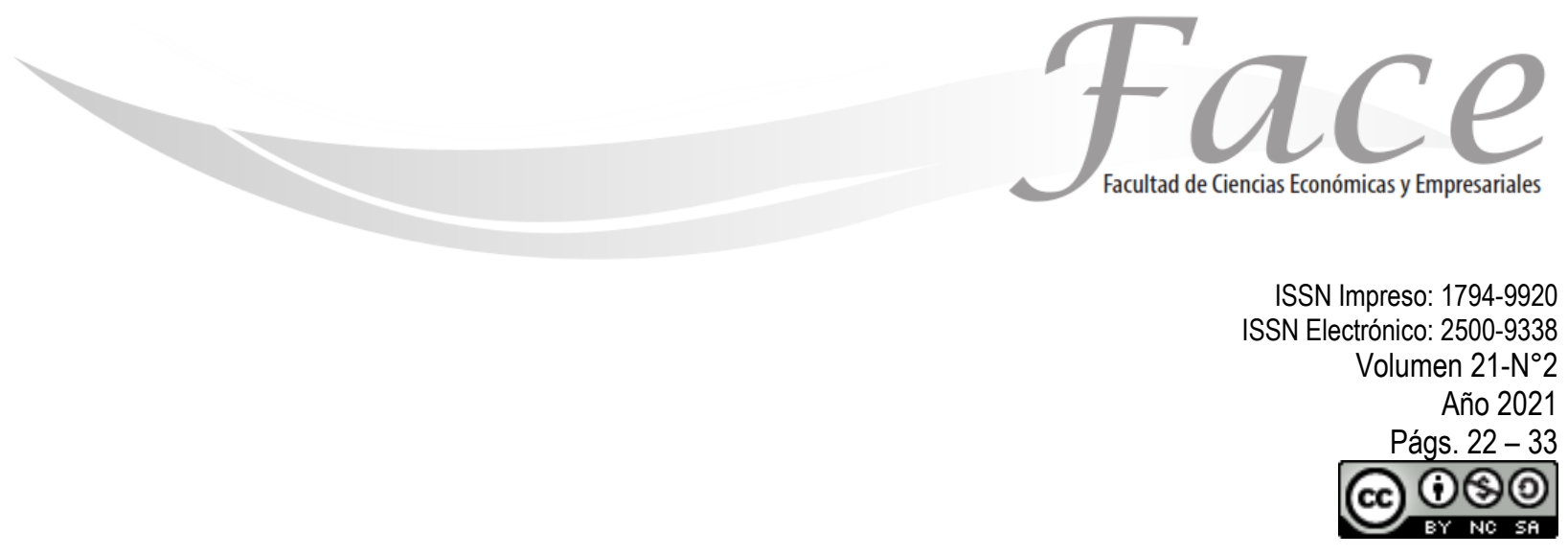

\title{
MERCADOTECNIA SOCIAL EN LA SALUD EN TIEMPOS DE COVID-19
}

\author{
Dra. Grace Aileen Ruiz Santoyo \\ Enlace ORCID: $\underline{0000-0002-0811-5131}$ \\ Dra. Berenice Juárez López \\ Enlace ORCID: $\underline{0000-0002-5261-3066}$ \\ Dra. Albany Aguilera Fernández \\ Enlace ORCID: 0000-0001-5506-2901
}

Fecha de Recepción: Febrero 11

Fecha de Aprobación: Mayo 23

\section{Resumen:}

Actualmente, las empresas y los gobiernos enfrentan serios problemas ocasionados por la pandemia del COVID-19, especialmente, el sector salud y el consumidor sanitario. Así mismo, la disciplina de la mercadotecnia ha tenido importantes cambios, sobre todo, el estudio de la mercadotecnia social en la salud. Las estrategias publicitarias se han adaptado a la nueva normalidad, mediante la difusión de mensajes en diversos canales de distribución, con el objetivo de concientizar a la población. Con base en lo anterior, el objetivo de la investigación es analizar la mercadotecnia social en la salud y proponer un modelo teórico a partir de las estrategias publicitarias, la difusión de los mensajes y los canales de distribución en el consumidor sanitario durante la pandemia. La metodología de investigación es descriptiva, con un enfoque causal y transversal, a mediante el diseño de un instrumento. Finalmente, se concluye que los gobiernos y las empresas deben realizar esfuerzos por desarrollar estrategias publicitarias de mercadotecnia social en la salud a partir de la difusión de mensajes y selección de canales de distribución adecuados para lograr la concientización de la población.

Palabras clave: marketing social, estrategias publicitarias, difusión de mensajes, canales de distribución.

* Profesora Investigadora, Facultad de Economía y Mercadotecnia , Universidad Autónoma de Coahuila. Ciudad Universitaria, Torreón- México. Contacto: grace.ruiz@uadec.edu.mx

** Profesora Investigadora, Facultad de Economía y Mercadotecnia , Universidad Autónoma de Coahuila. Ciudad Universitaria, Torreón- México. Contacto: berenice.juarez@uadec.edu.mx

${ }_{\star \star \star}$ Profesora Investigadora, Facultad de Economía y Mercadotecnia , Universidad Autónoma de Coahuila. México contacto: albany.aguilera@uadec.edu.mx 


\title{
SOCIAL MARKETING IN HEALTH IN TIMES OF COVID-19
}

\begin{abstract}
:
Currently, companies and governments face serious problems caused by the COVID-19 pandemic, especially the health sector and the health consumer. Likewise, the discipline of marketing has undergone important changes, especially the study of social marketing in health. Advertising strategies have been adapted to the new normal, through the dissemination of messages in various distribution channels, with the aim of raising awareness among the population. Based on the above, the objective of the research is to analyze social marketing in health and propose a theoretical model based on advertising strategies, the dissemination of messages and distribution channels in the health consumer during the pandemic. The research methodology is descriptive, with a causal and transversal approach, through the design of an instrument. Finally, it is concluded that governments and companies should make efforts to develop advertising strategies for social marketing in health based on the dissemination of messages and the selection of adequate distribution channels to raise awareness among the population.
\end{abstract}

Keywords: social marketing, advertising strategies, message dissemination, distribution channels.

\section{MARKETING SOCIAL EM SAÚDE NA ÉPOCA DA COVID-19}

\section{Resumo:}

Atualmente, empresas e governos enfrentam sérios problemas causados pela pandemia COVID-19, especialmente 0 setor de saúde e o consumidor de saúde. Da mesma forma, a disciplina de marketing passou por mudanças importantes, especialmente o estudo do marketing social na saúde. As estratégias publicitárias foram adaptadas ao novo normal, através da divulgação de mensagens nos diversos canais de distribuição, com o objetivo de sensibilizar a população. Com base no exposto, o objetivo da pesquisa é analisar o marketing social em saúde e propor um modelo teórico baseado em estratégias publicitárias, na disseminação de mensagens e nos canais de distribuição no consumidor de saúde durante a pandemia. A metodologia da pesquisa é descritiva, com abordagem causal e transversal, por meio da elaboração de um instrumento. Por fim, conclui-se que governos e empresas devem se empenhar no desenvolvimento de estratégias publicitárias de marketing social em saúde a partir da divulgação de mensagens e da seleção de canais de distribuição adequados para a conscientização da população.

Palavras-chave: marketing social, estratégias publicitárias, disseminação de mensagens, canais de distribuição. 


\section{INTRODUCCIÓN:}

A nivel mundial, y a principios de la década pasada, las micro, pequeñas y medianas empresas comenzaron a incursionar en el marketing digital y comercio electrónico, al plantear principalmente estrategias con contenido digital, debido a que los consumidores se hicieron mayormente dependientes de las plataformas y aplicaciones para sus actividades diarias (Kotler, Kartajaya y Setiawan, 2021).

A principios del año 2020, las empresas se vieron afectadas por la pandemia del COVID-19, por lo que enfrentan retos importantes para obtener ventas. Entre los problemas más destacados, se encuentra la adaptación de las organizaciones al aislamiento social $y$, sobre todo, a los nuevos hábitos de consumo por parte de la población. Expertos en economía, finanzas y negocios han afirmado que muchos sectores empresariales están y estarán muertos durante muchos años debido a la pandemia. Larios, Fischer, Peñalosa \& Ortega (2021). Sin embargo, la pandemia no sólo trajo dificultades para las empresas, sino también graves problemas económicos para los países. La crisis económica provocó que diversos indicadores macroeconómicos cambiarán en cuestión de meses.

En América Latina, se observaron niveles bajos de crecimiento económico en los países, lo que ocasionó el aumento de la pobreza y desigualdad social. Aunado a ello, el mercado laboral presentó pérdidas de empleo en diferentes sectores económicos. Lo anterior, se agravó especialmente a partir de la pandemia. Según las proyecciones de la Comisión Económica para América Latina y el Caribe (CEPAL), México es uno de los países que más se verá afectado en sus niveles de pobreza y pobreza extrema (CEPAL, 2020).

Entre los grupos vulnerables que han sido afectados por esta crisis económica, se encuentran los adultos mayores, ya que cerca de $13 \%$ de la población total en América Latina pertenece a dicho rango de edad (CEPAL, 2020). Los adultos mayores se caracterizan por tener una mortalidad más alta, especialmente ante el COVID, por lo que demandan mayor atención en los servicios de salud, tanto públicos como privados. Por lo anterior, los países han hecho esfuerzos por lograr el mejoramiento en sus políticas de salud, que permitan garantizar las mejores condiciones para dicho grupo vulnerable y para la población en general.
Ejemplo de lo anterior, son las campañas mundiales de salud desarrolladas por la Organización Mundial de la Salud (OMS), las cuales tienen por objetivo concientizar a la población en general sobre los problemas de salud que afectan a nivel local, regional, nacional e internacional. Entre sus campañas se encuentra el día mundial del lavado de manos, de la salud mental, del sida, de la hepatitis, de la seguridad del paciente, de la tuberculosis, de la salud, del paludismo, del donante de sangre, entre otras (OMS, 2021).

En México, la Secretaría de Salud (2010) elaboró un manual sobre mercadotecnia social en la salud, cuyo objetivo es la aplicación e incorporación de programas de salud pública en el país, así como el fortalecimiento, la creación e implementación de estrategias diseñadas para motivar los cambios en el comportamiento de la población.

En ese sentido, se emplearon diversos canales de distribución para la difusión de mensajes, con el fin de concientizar y cambiar el comportamiento de la población. El mensaje más destacado fue "quédate en casa", el cual especificaba la importancia del distanciamiento físico y aislamiento social de toda la ciudadanía, con el objetivo de proteger a los grupos vulnerables, quienes fueron considerados como población de riesgo.

Las campañas publicitarias tuvieron que desarrollarse a través de canales digitales, debido a la dependencia de la población por el uso de medios online, sin embargo, se desconoce si dichas campañas lograron ser exitosas en la población. Por ello, surge la interrogante, ¿las estrategias publicitarias, la difusión de los mensajes y los canales de distribución son factores clave para la mercadotecnia social en la salud?

Con base en lo anterior, el objetivo de la presente investigación es analizar la mercadotecnia social en la salud y proponer un modelo teórico a partir de las estrategias publicitarias, la difusión de los mensajes y los canales de distribución en el consumidor sanitario durante la pandemia. 


\section{MARCO TEÓRICO}

Los servicios de salud han evolucionado gracias a la investigación científica, el desarrollo tecnológico y la innovación. Las mayores inversiones se observan en el sector farmacéutico, sin embargo, diversos estudios señalan que la inteligencia artificial será una gran área de oportunidad para la medicina, ya que tiene el poder de transformar la atención médica (Kotler, Kartajaya y Setiawan, 2021),

Ejemplo de lo anterior, son algunas empresas que ofrecen atención médica y se centran en promover estilos de vida saludables y proporcionar herramientas de diagnóstico y medicamentos accesibles para toda la población. En el caso del COVID-19, el deseo de evitar la enfermedad se representa como un valor y la expectativa es el cambio en el comportamiento de la población, con la finalidad de prevenir o disminuir la posibilidad de contagio. Cabe señalar que las percepciones juegan un rol muy importante respecto a los problemas de salud, debido a las creencias personales que se tienen relacionadas con ciertas enfermedades. Por ello, la presente investigación tiene un enfoque desde la mercadotecnia social en la salud.

\section{Mercadotecnia social}

La mercadotecnia social ha cobrado relevancia durante la pandemia, debido a que ha sido utilizada como herramienta para la prevención y control del COVID-19. A nivel internacional, se observan países donde la mercadotecnia social ha puesto en práctica estrategias con el fin de cambiar el comportamiento, tanto de los gobiernos como de las empresas. Cabe señalar que los objetivos de la mercadotecnia social son proporcionar información, estimular acciones beneficiosas para la sociedad, cambiar comportamientos nocivos, cambiar los valores de la sociedad (Góngora, 2014).

De igual manera, la mercadotecnia social se concentra en ideas, actitudes y comportamientos para el beneficio de la sociedad en general. Así mismo, se dirige a las personas con la finalidad de orientarlas a aceptar, rechazar 0 modificar una conducta determinada que pone en riesgo su salud, calidad de vida, bienestar y/o seguridad. Su propósito es crear conciencia, cambiar el comportamiento y lograr aceptación de una conducta deseada.
La mercadotecnia en salud consta de dos dimensiones, la comercial y la social. La primera tiene un interés económico y la segunda busca mejorar la calidad de vida bajo un enfoque ético y colectivo, que pretende incrementar la aceptación y concientización de una idea, con el objetivo de inducir cambios en el comportamiento para mejorar la vida de las personas. En otras palabras, la mercadotecnia social tiene como objetivo cambiar o transformar un comportamiento dañino y adoptar nuevas ideas saludables (MezaSánchez, Orozco-Rodríguez, Martínez-Acosta, y Viramontes-Martínez, 2021).

En ese sentido, las personas buscan mejores condiciones de vida, lo que incluye el acceso a la educación, salud y seguridad pública. Según Giuliani, Monteiro, Zambon, Betanho y Lima (2012), muchas empresas buscan desarrollar acciones que las hagan más conocidas y aceptadas por el público, por lo que se concentran en procesos de marketing para alcanzar los resultados esperados y que estén de acuerdo con las expectativas sociales colectivas.

En casos parecidos, la mercadotecnia social se vuelve fundamental para las empresas, ya que las relaciones entre los consumidores y las organizaciones están cada vez más desarrolladas. El estudio anterior, señala que las campañas sociales buscan cambiar el comportamiento de las personas, por lo tanto, la mercadotecnia social se refiere a una manera de influir a favor de la aceptación de ideas sociales relevantes (Giuliani et al. 2012).

De esa manera, la confianza generada en la mercadotecnia social puede llevar a que sea entendida como una herramienta con potencial para impulsar transformaciones sociales significativas. Es decir, la mercadotecnia social puede emplearse para las acciones de apoyo a diversas causas sociales y no sólo para lograr que las empresas se promuevan a sí mismas.

\section{Mercadotecnia social en la salud}

La mercadotecnia social en la salud se define como el desarrollo de estrategias que motivan el cambio de hábitos, actitudes, acciones, comportamientos, conductas, valores o prácticas para mejorar la salud de los individuos y las comunidades, mediante la 
investigación de las necesidades, así como la planeación, ejecución y comunicación como herramientas estratégicas (Secretaría de Salud, 2010).

Los programas o campañas de mercadotecnia social en la salud lograrán el éxito a partir de una profunda investigación sobre las necesidades, deseos, creencias y actitudes específicas del público objetivo, así como de las características concretas de los productos y de la forma en que los perciben los consumidores. Cabe señalar que es necesario contar con una metodología de investigación que permita planificar las estrategias acertadas, las cuales deben comprender la toma de decisiones por parte de las personas (Góngora, 2014).

Actualmente, las campañas de salud pública son una gran oportunidad para aumentar la concientización y los conocimientos sobre los problemas de salud, así como, para movilizar apoyos en todos los ámbitos a nivel local, regional, nacional e internacional. Un ejemplo, es el consumo sanitario durante el COVID-19.

Tabla 1.

Mezcla de la mercadotecnia social en la salud

\begin{tabular}{ll}
\hline Producto básico & Disminuir la posibilidad de contagio del COVID-19. \\
Producto deseado & Aislarse y mejorar el sistema inmunológico. \\
Producto aumentado & $\begin{array}{l}\text { Informarse en centros especializados, a través de líneas } \\
\text { digitales. }\end{array}$ \\
Beneficios & Mejorar la calidad de vida. \\
\hline
\end{tabular}

Fuente: Elaboración propia.

Para el logro de la mezcla anterior, es necesaria la promoción mediante el uso integrado de estrategias de comunicación, así como elaborar y definir los mensajes a transmitir y los canales de distribución. En ese sentido, se debe especificar lo que se va a divulgar, para influenciar en los grupos objetivos, con el objetivo de que aprendan, crean, cambien el comportamiento y adopten la conducta deseada. Así mismo, la difusión de dichos mensajes debe realizarse a través de medios masivos electrónicos, de visibilidad externa, impresos, comunicación cara a cara y medios complementarios. Finalmente, puntualizar los canales donde se difundirá la información.

\section{Antecedentes de investigación}

Diversos autores han analizado la promoción de la salud a través de diversos canales de distribución. Pérez-Moedano, Godínez-Viggiano, Hernandez-Pérez y Ricaño-Olarte (2021), analizaron 40 campañas audiovisuales, de las cuales $80 \%$ provenían del sector público y $20 \%$ del sector privado. Los resultados de dicha investigación resaltan la difusión de mensajes por parte de la Secretaría de Salud en México, donde la población identificó, claramente, la información relacionada con los cuidados de salud.

Por otro lado, empresas han adaptado sus estrategias publicitarias a las condiciones de pandemia. Ejemplo de ello, es la redefinición de los objetivos comerciales, difusión de mensajes para las comunicaciones críticas, utilización de diversos canales de comunicación, promociones de emergencia, capacitación de los empleados, precios, consignación y el llamado "marketing coronavirus".

Según Meza-Sánchez et al (2021), los siguientes factores demuestran la relación existente entre las campañas y los modelos de salud:

1. El lavado de manos.

2. Evitar el contacto público.

3. Distanciamiento social.

4. Uso de mascarillas.

5. Respetar cuarentena de ser necesario.

Estos cince factores han demostrado ser necesarios para la mercadotecnia social en la salud. De igual manera, se ha observado la relevancia de brindar información verídica, científicamente confirmada, para evitar rumores, que a través de las redes sociales se propagan con gran inmediatez y propician el no deseado pánico en la población (Suárez, 2020).

Con base en la investigación de Suárez (2020), el mensaje a difundir es: "Todos estamos expuestos, la prevención personal es la única alternativa viable" y los canales de distribución se ha realizado por los medios de comunicación y redes sociales, así mismo, se han empleado líderes de opinión y científicos destacados, en todas las ramas de la ciencia, quienes ofrecen información.

Elegir los canales de distribución del mensaje adecuados, facilitará crear estrategias publicitarias que hagan conciencia ante el COVID-19, debido a que en esta etapa se eligen los medios para la difusión del mensaje, donde cobra importancia el papel del público objetivo, ya que es necesario conocer dónde se ubica, qué piensa y, sobre todo, cómo percibe la difusión de 
los mensajes a través de las estrategias publicitarias de mercadotecnia social en la salud.

En ese sentido, la difusión de los mensajes y los canales de distribución deben adaptarse al entorno y contexto en el que se encuentre el consumidor sanitario, debido a que existe una relación directa entre estas variables con la selección del público objetivo. Dicho de otra manera, es necesario un plan de mercadotecnia que cumpla con objetivos, tácticas, canales y, en general, la opinión de los consumidores.

\section{Estrategias publicitarias y difusión de mensajes}

Diversas investigaciones han relacionado las estrategias publicitarias y la difusión de los mensajes. El estudio de Rando (2020), señala que existe una brecha entre los objetivos los centros sanitarios y las actividades realizadas en redes sociales. Así mismo, mencionan que dicha información no es fiable, debido a la falta de coincidencia entre los objetivos de los mensajes que se quieren comunicar y las estrategias publicitarias de las entidades sanitarias.

Las recomendaciones de dicha investigación giran en torno a que las estrategias publicitarias deben ir encaminadas a la imagen corporativa y la imagen de los profesionales de salud, así como la difusión de mensajes a partir de temas sobre salud en general, promoción de hábitos de vida saludables, investigación y noticias sobre la actividad del hospital (Rando, 2020).

Los autores Holden, Nanayakkara, Skinner, Spallek y Sohn (2021), realizaron un estudio en Australia a partir de la aplicación de una encuesta, donde demostraron que muchos consumidores de productos sanitarios sentían que, si bien las estrategias publicitarias sanitarias eran útiles, el mensaje fue confuso, debido a que muchos participantes tenían creencias erróneas sobre algunos elementos de la publicidad sanitaria. En este sentido, se observó cómo la difusión del mensaje no siempre llega de manera correcta a la población.

El cuestionario se integró por preguntas específicas sobre la confusión que generaba la publicidad de algunos servicios de salud para las necesidades personales; así mismo, se cuestionó la diferencia entre distinguir los servicios que mejoran la salud y los que mejoran la parte cosmética; por último, se discutió acerca de si las estrategias publicitarias ayudan a la difusión de los mensajes sobre los servicios de salud disponibles (Holden et al. 2021).

Con base en lo anterior, se genera la primera hipótesis de investigación:

H1: Las estrategias publicitarias se relacionan con la difusión de los mensajes en la mercadotecnia social en la salud en tiempos de COVID-19.

\section{Estrategias publicitarias y canales de distribución}

La gestión de la comunicación que llevan a cabo algunas instituciones sanitarias, a través de los diversos canales de distribución, permiten elaborar estrategias publicitarias para la mejora en la calidad de vida y bienestar de los consumidores sanitarios. Las estrategias publicitarias pueden estar orientadas a mejorar la eficacia de la comunicación entre la entidad sanitaria y los usuarios directos de los sistemas sanitarios públicos y privados (Ferreira, 2021).

Los sitios web cobraron relevancia durante la pandemia, debido a que permitieron a los consumidores sanitarios contar con una fuente de información útil y confiable. De igual manera, tomaron importancia las recomendaciones de boca en boca, tanto de familiares, amigos, líderes de opinión y profesionales de la salud, gracias a que la población logró identificar los mensajes y brindar la confianza a las diversas reseñas y testimonios en línea (Holden et al. 2021).

Las estrategias publicitarias deben estar relacionadas con los canales de distribución. En España, se desarrolló un estudio donde se contempló que las redes sociales son el mejor canal para adaptarse a los consumidores sanitarios, así mismo, dichas redes son las más conocidas, de mayor impacto y con mayor número de seguidores. Por lo tanto, se vuelve ideales para informar, interactuar y crear comunidad, así como las de mayor sencillez y rapidez de respuesta (Rando, 2020).

De igual manera, el trabajo de Masip, AranRamspott,Ruiz-Caballero, Suau, Almenar y PuertasGraell (2020), aplicó un cuestionario en España, donde se analizaron las variables de consumo de noticias durante el confinamiento, uso de redes sociales, confianza en los medios de comunicación, desinformación y noticias falsas. Los hallazgos de esta 
investigación afirman que los medios digitales fueron la primera opción para informarse, seguidos por la televisión, las redes sociales y las aplicaciones de mensajería instantánea, sin embargo, si se suman los porcentajes de las tres opciones, los informativos de televisión lideran el ranking como medio preferido para informarse.

En ese sentido, la investigación de Masip et al. (2020), muestra datos diferentes a los trabajos de Rando (2020), ya que la primera concluye que los canales de distribución, mayormente usados, son los medios tradicionales, y que sólo la mitad de los sujetos de estudio prefieren la difusión de mensajes respecto al COVID-19. Por ello, se vuelve interesante conocer el comportamiento sanitario en otros países.

Con base en lo anterior, se genera la segunda hipótesis de investigación:

H2: Las estrategias publicitarias se relacionan con los canales de distribución. en la mercadotecnia social en la salud en tiempos de COVID-19.

\section{Difusión de mensajes y canales de distribución}

Las campañas de mercadotecnia social deben incluir comunicaciones múltiples, debido a que la naturaleza de la mercadotecnia social se centra en que la audiencia proporciona un marco eficaz, para aumentar la adopción de mensajes, lo anterior, con el objetivo de influir en el cambio de comportamiento del consumidor sanitario. Respecto a los canales de distribución, investigaciones concluyen que los medios digitales, específicamente las redes sociales, son una forma rentable y adecuada de aumentar el alcance de las estrategias publicitarias (Sundstrom, DeMaria, Ferrara, Meier, Vyge, Billings, DiBona y McLernon Sykes, 2021).

La difusión de los mensajes y los canales de distribución muestran una relación de dependencia, ya que existe una asociación entre el tipo de medio elegido y la adherencia al contenido de información sobre el coronavirus. Ferreira (2021), aplicó un cuestionario en Portugal, donde analizó la difusión de los mensajes y el canal de información, cuyos resultados de dicho estudio revelaron la existencia de un fenómeno de dependencia entre los medios de comunicación convencionales (televisión y periódicos digitales), con una fuerte exposición a los contenidos informativos, y el contenido de los mensajes.
Dentro de las investigaciones más destacas en temas de salud, se encuentra la de Nielsen, Fletcher, Newman, Brennen y Howard (2020), quienes aplicaron una encuesta en línea en los países de Argentina, Alemania, Corea del Sur, España, Reino Unido y Estados Unidos. En algunos países encontraron que el canal de distribución preferido por la población, para obtener información sobre el COVID-19, fueron las redes sociales, sitios de videos y aplicaciones de mensajería. Así mismo, la difusión de los mensajes permitió la concientización y comprensión de la situación sanitaria a nivel mundial. Finalmente, a excepción de Argentina y España, se estimó una relación positiva y significativa entre la fuente de información y el mensaje sobre el conocimiento del coronavirus.

Con base en lo anterior, se genera la tercera hipótesis de investigación:

H3: La difusión de los mensajes se relacionan con los canales de distribución en la mercadotecnia social en la salud en tiempos de COVID-19.

\section{METODOLOGÍA}

\section{Modelo}

Con base en la revisión de la literatura, así como la evidencia empírica internacional, se plantea la propuesta de un modelo de investigación, el cual representa las relaciones entre las variables. En ese sentido, la propuesta de modelo teórico de investigación quedó integrado de la siguiente manera: El factor 1, estrategias publicitarias, se compone de tres variables, concientización, responsabilidad social y financiamiento; el factor 2, difusión de mensajes, cuenta con tres variables, lenguaje, material y videos/anuncios; el factor 3, canales de distribución, tiene dos variables: sitios y medios tradicionales 0 digitales. En su totalidad, el modelo se compone de ocho variables. 
Figura 1.

Modelo de la mercadotecnia social en la salud

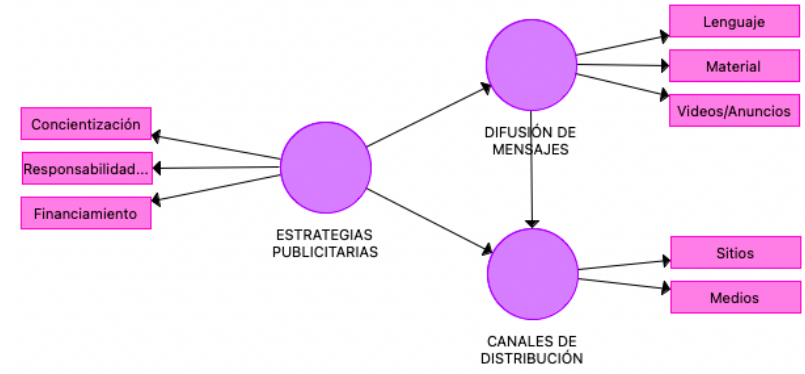

Fuente: Elaboración propia con base en Masip et al. (2020), Holden et al. (2021), Rando (2020), Ferreira (2021) y Nielsen (2020).

La delimitación espacial de la presente investigación es la Región Laguna, la cual comprende el área geográfica resultante de una fusión de las ciudades de Gómez Palacio y Lerdo, por parte del estado de Durango, y de Torreón y Matamoros, por parte del estado de Coahuila de Zaragoza. Esta región está posicionada como la novena zona metropolitana más poblada de México, por debajo de la región de Ciudad Juárez y El Paso, y por arriba de la región de Querétaro (INEGI, 2016). Cabe destacar, que la entidad federativa de Coahuila fue uno de los dos estados en comenzar con la campaña de vacunación contra el COVID-19, gracias a la logística a cargo del Ejército Nacional y el cumplimiento de las condiciones de refrigeración que requiere.

Con base en lo anterior, la presente investigación cobra relevancia, puesto que para la Región Laguna y zonas metropolitanas que comparten características, existe escasa evidencia empírica sobre la mercadotecnia social en la salud. Por ello, una de las aportaciones de esta investigación es brindar antecedentes que sean de utilidad para las instituciones de seguridad social y empresas interesadas en promover campañas de mercadotecnia social.

La metodología propuesta es cuantitativa, la cual se utiliza cuando se plantea un problema delimitado y concreto (Hernández, Fernández y Baptista, 2010). Por ello, esta investigación teórica es de tipo descriptiva y tiene un enfoque causal y transversal. El sujeto de estudio será la población de 16 años en adelante que padezca alguna enfermedad crónica degenerativa como diabetes, enfermedades respiratorias y/o cardiacas, entre otras. La recolección de la información se llevará a cabo mediante el diseño de un cuestionario en línea y aplicado mediante la plataforma Google Forms.

Tabla 2. Ficha técnica de investigación

\begin{tabular}{ll}
\hline Universo & $\begin{array}{l}\text { Hombres y mujeres con alguna } \\
\text { enfermedad crónica degenerativa } \\
\text { en la Región Laguna. }\end{array}$ \\
$\begin{array}{l}\text { Tamaño } \\
\text { muestra }\end{array}$ & 385 observaciones. \\
Error muestra & $5 \%$ \\
Nivel de & $95 \%$ \\
confianza & \\
$\begin{array}{l}\text { Procedimiento } \\
\text { de muestreo }\end{array}$ & Muestreo aleatorio simple. \\
$\begin{array}{l}\text { Procesamient } \\
\text { de la }\end{array}$ & SPSS Statistics $@ 25$ y SmartPLS 8 \\
información & 3.3 .3$.
\end{tabular}

Fuente: Elaboración propia.

En la tabla anterior se muestra la ficha técnica de investigación, la cual especifica la población y muestra, así como la información para la obtención de los datos. Cabe señalar que, para la presente investigación, únicamente se realizó la validación de contenido, debido a que el instrumento se encuentra en proceso de aplicación.

\section{Instrumento}

Como parte de los objetivos de investigación, se obtuvo el diseño del instrumento y la validez del contenido de este. A continuación, se presenta el cuestionario, el cual está integrado por tres variables y ocho ítems, los cuales fueron medidos por medio de una escala tipo Likert de 7 puntos que osciló entre $1=$ nada importante y $7=$ muy importante. 
ISSN: 1794-9920 Impreso / Electrónico 2500-9338

Junio - Diciembre. Volumen 21 Número 2, Año 2021 Págs. 22-33

Tabla 3.

Instrumento de mercadotecnia social en la salud

Desde su perspectiva, ante la llegada de la pandemia del COVID-19 y, con ello, las campañas sociales de salud implementadas para el cuidado y prevención de este virus. Califique las siguientes preguntas del 1 al 7 , donde 1 es "nada importante" y 7 "muy importante".

Estrategias ¿Qué tan importantes son las estrategias publicitarias publicitarias de mercadotecnia social para su forma de pensar y actuar (concientización)?

¿Qué tan importante es la responsabilidad social en las estrategias publicitarias emitidas por las campañas de salud?

¿Qué tan importante es el financiamiento en las estrategias publicitarias emitidas por las campañas de salud?

Difusión de ¿Qué tan importante es la formalidad del lenguaje mensajes usado en los programas sociales para generar confianza en usted?

¿Qué tan importante es el material creativo/amigable usado en los programas sociales para generar confianza en usted?

¿Qué tan importante es la difusión de videos/anuncios promocionales para generar confianza en usted?

Canales de ¿Qué tan importante es el sitio (páginas, redes distribución sociales, canales, etc.) para ayudar a crear conciencia en usted?

¿Qué tan importante es el vínculo (empatía) que tiene con los canales de distribución (medios tradicionales y digitales) para ayudar a crear conciencia en usted?

Fuente: Elaboración propia.

Cabe mencionar que, para la elaboración del cuestionario anterior, se utilizaron escalas de medición testadas en otras investigaciones a nivel internacional.

\section{Validez de contenido}

La validación de mensajes se realiza con los representantes de un grupo de personas, ya sea el público objetivo o grupos técnicos, con la finalidad de conocer su percepción y opinión respecto a la comprensión de las preguntas, antes de iniciar su difusión. Diversas investigaciones utilizan la técnica cualitativa de entrevista para la validación, con el objetivo de obtener información con mayor grado de profundidad y conocimiento especializado.

El proceso de validación se desarrolla a partir de la validez de contenido, la cual se llevó a cabo con base en la opinión de expertos y/o especialistas en el tema, quienes cuentan con un dominio del contenido teórico de cada elemento de análisis, de tal manera que sus comentarios permitieron obtener la congruencia teórica del instrumento de evaluación.
Se entrevistó a cuatro profesores de mercadotecnia, quienes tuvieron la oportunidad de revisar el instrumento a profundidad y realizar comentarios $u$ observaciones, con la intención de mejorar los aspectos de contenido, redacción y sintaxis del cuestionario.

Dentro de los señalamientos, se destacan las siguientes sugerencias:

- Mantener el mismo diseño o formato.

- Homologar la escala de medición.

- Agregar, en estrategias publicitarias, responsabilidad social.

- Eliminar, en canales de distribución, no sólo para población vulnerable.

- Observar, en canales de distribución, que sólo son dos ítems.

- Revisar el orden de las preguntas.

Con base en lo anterior, se hicieron las correcciones al instrumento y se obtuvo una nueva versión del cuestionario. Cabe mencionar que, una vez realizada la validez de contenido, será necesaria la obtención de la información con el objetivo de que los datos permitan realizar las siguientes estimaciones, sin embargo, se puede adelantar sobre la validez de constructo, la cual se efectuará al corroborar cada ítem con la dimensión a la que corresponda, según el factor, a partir de diversas pruebas estadísticas.

Finalmente, la validez del criterio se realizará al medir el instrumento y demostrar que existen relaciones teóricas con otros métodos. Es necesario mencionar que el modelo teórico propuesto de investigación es una aproximación a la realidad, el cual es una proposición que deberá ser corroborada hasta que se logren aplicar las diversas pruebas de hipótesis.

\section{RESULTADOS:}

Como resultados parciales de la investigación, se obtuvo el análisis de la mercadotecnia social en la salud, la propuesta de un modelo teórico, a partir de la revisión de literatura y el sustento teórico de las variables para el diseño del instrumento. Sin embargo, al momento del envío de este trabajo, el cuestionario se encuentra en la fase de aplicación. 


\section{DISCUSIÓN Y CONCLUSIONES}

El objetivo inicial de esta investigación era analizar la mercadotecnia social en la salud y proponer un modelo teórico a partir de las estrategias publicitarias, la difusión de los mensajes y los canales de distribución en el consumidor sanitario durante el COVID-19. En ese sentido, se afirma que el modelo teórico propuesto se basa en la evidencia empírica internacional. Así mismo, se observa que existe una relación entre los factores estrategias publicitarias, difusión de mensajes y canales de distribución, las cuales, a su vez, son medidos por las variables concientización, responsabilidad social, financiamiento, lenguaje, material, videos/anuncios, sitios y medios tradiciones 0 digitales. Lo anterior, siempre y cuando se clasifique de manera correcta al público objetivo, ya que teniendo claro esto, el mensaje creará un impacto social relevante.

Las recomendaciones preliminares se desarrollan con base en las implicaciones gerenciales $y$ gubernamentales. En el caso de las empresas, se recomienda realizar esfuerzos para la interacción en medios digitales de comunicación, para conocer el mercado y dar respuesta de forma personalizada a través del correo electrónico o redes sociales. Así mismo, se sugiere segmentar campañas y adaptar la difusión de mensajes en función de las empresas, así como recompensar la interacción de los usuarios con diversas promociones. De igual manera, elegir los canales de distribución del mensaje con base en las características particulares de los productos y/o servicios que se tienen disponibles.

En el caso del sector público, las recomendaciones se enfocan en aumentar la difusión de mensajes sobre los cuidados en la salud, los problemas y soluciones, con el objetivo de influir en las actitudes para producir cambios en el comportamiento de la población. De la misma forma, las estrategias pueden ir encaminadas a demostrar o ejemplificar habilidades saludables, reforzar conocimientos, actitudes 0 conductas, enseñar el beneficio del cambio de la conducta, así como descartar mitos y noticias falsas.

En conclusión, la información que brinda la mercadotecnia social en la salud muestra una clara necesidad en primer instancia de contar con antecedentes que sean de utilidad para formular $y$ desarrollar estrategias publicitarias que permitan pensar y actuar con base en la responsabilidad social por parte de las instituciones y empresas interesadas en promover campañas de mercadotecnia social, a través de la difusión de mensajes con material amigable, creativo, dinámico y lenguaje claro para lograr la concientización de la población, así como la elección adecuada de los canales de distribución que permitan vincular al público objetivo.

Finalmente, cabe aclarar que las hipótesis planteadas en el modelo teórico propuesto serán probadas en una segunda etapa de la investigación, a partir de estimar la fiabilidad y validez del instrumento, así como el análisis factorial confirmatorio y el análisis de los datos por medio de la técnica estadística de regresión de mínimos cuadrados parciales.

\section{REFERENCIAS:}

CEPAL (2020), El desafío social en tiempos del COVID-19, Informe Especial No.3 de la Comisión Económica para América Latina y el Caribe, Recuperado de https://repositorio.cepal.org/bitstream/handle/ 11362/45527/5/S2000325 es.pdf

Ferreira, G.B. (2021), Quando as notícias importam. Fontes, confiança e desinformação em tempos de Covid-19. En: Rui, F. y Ferreira, I. Perspectivas multidisciplinares da Comunicação em contexto de Pandemia. Lisboa: Coleção ICNOVA. https://www.icnova.fcsh.unl.pt/perspectivasmultidisciplinares-da-comunicacao-emcontexto-de-pandemia-vol-i/ 
Giuliani, A. C., Monteiro, T. A., Zambon, M. S., Betanho, C. y Lima, L. H. (2012), El marketing social, el marketing relacionado a causas sociales y la responsabilidad social empresarial: El caso del supermercado Pãode-Açucar, de Brasil. Invenio, 15(29), 11-27. https://www.redalyc.org/articulo.oa?id=87724 146003

Góngora, L. H. (2014), Mercadotecnia social: una herramienta necesaria para la promoción de la salud. Medisan, 18(5), 684-694. http://scielo.sld.cu/scielo.php?script=sci_abst ract\&pid=S1029$30192014000500012 \&$ lng=es\&nrm=iso

Hernández, R., Fernández, C. y Baptista, M. P. (2010), Metodología de la investigación. Ciudad de México, México: McGraw-Hill.

Holden, A.C.L., Nanayakkara, S., Skinner, J., Spallek, H. y Sohn, W. (2021), What do Australian health consumers believe about commercial advertisements and testimonials? a survey on health service advertising. BMC Public Health, 21(74), 1-12. https://doi.org/10.1186/s12889-020-10078-9

INEGI, (2016) Banco de Información Económica. Ciudad de México: Instituto Nacional de Estadística y Geografía. Recuperado de https://www.inegi.org.mx/app/indicadores/bie. $\underline{\mathrm{html}}$

Kotler, P., Kartajaya, H. y Setiawan, I. (2021), Marketing 5.0: Technology for Humanity. Hoboken, New Jersey: Wiley.

Larios-Gómez E, Fischer L, Peñalosa M, OrtegaVivanco M. Purchase behavior in COVID-19: A cross study in Mexico, Colombia, and Ecuador. Heliyon. 2021 Mar 25;7(3):e06468. doi: 10.1016/j.heliyon.2021.e06468. PMID: 33855230; PMCID: PMC8027689
Masip, P., Aran-Ramspott, S., Ruiz-Caballero, C., Suau, J., Almenar, E. y Puertas-Graell, D. (2020), Consumo informativo y cobertura mediática durante el confinamiento por el Covid-19: sobreinformación, sesgo ideológico y sensacionalismo. El profesional de la información, 29(3). https://doi.org/10.3145/epi.2020.may.12

Meza-Sánchez, D. L., Orozco-Rodríguez, A. Y., Martínez-Acosta, E. G. y ViramontesMartínez, L. M. (2021), Revisión teórica de la mercadotecnia en salud y su importancia en la pandemia de COVID-19. Journal of Basic and Applied Psychology Research, 2(4), 0713.

https://repository.uaeh.edu.mx/revistas/index. php/jbapr/issue/archive

Nielsen, R.K., Fletcher, R., Newman, N., Brennen, J.S. y Howard, P.N. (2020), Navigating the 'Infodemic': How People in Six Countries Access and Rate News and Information about Coronavirus. Oxford: Reuters Institute for the Study of Journalism.

https://reutersinstitute.politics.ox.ac.uk/infode mic-how-people-six-countries-access-andrate-news-and-information-about-coronavirus

OMS (2021), Campañas mundiales de salud pública de la Organización Mundial de la Salud, Recuperado de https://www.who.int/es/campaigns

Pérez-Moedano, K. X., Godínez-Viggiano, M. E., Hernandez-Pérez, H. A. y Ricaño-Olarte, A. (2021), Salud Pública en tiempos de COVID19: Un análisis de las campañas audiovisuales. Journal of Basic and Applied Psychology Research, 2(4), 20-26. https://repository.uaeh.edu.mx/revistas/index. php/jbapr/issue/archive

Rando, D. (2020), Eficacia de la comunicación sanitaria vía redes sociales, Tesis de doctorado, Universidad de Málaga. https://riuma.uma.es/xmlui/handle/10630/212 $\underline{30}$ 
Secretaría de Salud (2010), Manual de mercadotecnia social en salud, Recuperado de https://docplayer.es/260280-Secretaria-desalud-manual-de-mercadotecnia-social-ensalud.html

Suárez, N. (2020), Mercadotecnia social en la prevención del coronavirus. Horizonte sanitario, 19(3), 307-309.

https://revistas.ujat.mx/index.php/horizonte

Sundstrom, B., DeMaria, A. L., Ferrara, M., Meier, S., Vyge, K., Billings, D., DiBona, D. y McLernon Sykes, B. M. (2021). You Have Options: Implementing and evaluating a contraceptive choice social marketing campaign. Medicine Access @ Point of Care, 5, 1-10. https://doi.org/10.1177/23992026211003499 